\title{
Patterns of recurrence and second primary lung cancer in early-stage lung cancer survivors followed with routine computed tomography surveillance
}

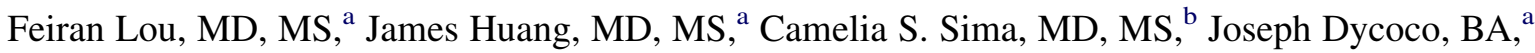 \\ Valerie Rusch, MD, ${ }^{\mathrm{a}}$ and Peter B. Bach, MD, MAPP
}

\begin{abstract}
Objective: At present, there is no consensus on the optimal strategy for follow-up care after curative resection for lung cancer. We sought to understand the patterns of recurrence and second primary lung cancer, and their mode of detection, after resection for early-stage non-small cell lung cancer in patients who were followed by routine surveillance computed tomography scan.
\end{abstract}

\begin{abstract}
Methods: We reviewed the outcomes of consecutive patients who underwent resection for early-stage nonsmall cell lung cancer at Memorial Sloan-Kettering Cancer Center between 2004 and 2009.

Results: A total of 1294 consecutive patients with early-stage non-small cell lung cancer underwent resection. The median length of follow-up was 35 months. Recurrence was diagnosed in 257 patients $(20 \%)$, and second primary lung cancer was diagnosed in 91 patients $(7 \%)$. The majority of new primary cancers $(85[93 \%])$ were identified by scheduled routine computed tomography scan, as were a smaller majority of recurrences (157 $[61 \%])$. During the first 4 years after surgery, the risk of recurrence ranged from $6 \%$ to $10 \%$ per person-year but decreased thereafter to $2 \%$. Conversely, the risk of second primary lung cancer ranged from $3 \%$ to $6 \%$ per person-year and did not diminish over time. Additional testing after false-positive surveillance computed tomography scan results was performed for 329 patients $(25 \%)$, but only 4 of these patients $(0.3 \%)$ experienced complications as a result of subsequent invasive diagnostic procedures.
\end{abstract}

Conclusions: Almost all second primary cancers and the majority of recurrences were detected by posttherapeutic surveillance computed tomography scan. The risk of recurrence for early-stage non-small cell lung cancer survivors persisted during the first 4 years after resection, and vigilance in surveillance should be maintained. (J Thorac Cardiovasc Surg 2013;145:75-82)

Lung cancer is an aggressive malignancy that remains the leading cause of cancer-related death in the United States. ${ }^{1}$ Its poor prognosis has been attributed to late detection and its high rate of relapse despite attempts at curative treatment. Continued follow-up after completion of therapy is crucial to monitor for complications, recurrences, and second primary malignancies, and new disease would ideally be detected as early as possible. Low-dose computed tomography (CT) screening of high-risk patients recently has been shown to significantly decrease lung cancer-

\footnotetext{
From the Thoracic Service, ${ }^{\mathrm{a}}$ Department of Surgery, Biostatistics Service, ${ }^{\mathrm{b}}$ Department of Epidemiology and Biostatistics, and Health Outcomes Service, ${ }^{\mathrm{c}}$ Department of Epidemiology and Biostatistics, Memorial Sloan-Kettering Cancer Center, New York, NY.

Disclosures: Authors have nothing to disclose with regard to commercial support.

Read at the 38th Annual Meeting of The Western Thoracic Surgical Association, Maui, Hawaii, June 27-30, 2012.

Received for publication May 25, 2012; revisions received Aug 31, 2012; accepted for publication Sept 13, 2012; available ahead of print Nov 5, 2012.

Address for reprints: James Huang, MD, MS, Thoracic Service, Department of Surgery, Memorial Sloan-Kettering Cancer Center, 1275 York Ave, New York, NY 10065 (E-mail: huangj@mskcc.org).

$0022-5223 / \$ 36.00$

Copyright (C) 2013 by The American Association for Thoracic Surgery

http://dx.doi.org/10.1016/j.jtcvs.2012.09.030
}

related mortality, compared with chest x-ray screening. ${ }^{2}$ However, the use of routine imaging for the surveillance of lung cancer survivors has not been well defined. As a result, surveillance guidelines and practices vary greatly in imaging frequency and modality. ${ }^{3}$ The interpretation of previous studies is limited by their small sample sizes and by the heterogeneity of their surveillance methods. Although some studies have suggested that surveillance CT scan is effective at detecting early second primary lung cancer, ${ }^{4}$ the data are less clear regarding detection of recurrence. Some studies report that routine surveillance fails to identify most asymptomatic recurrences. ${ }^{5}$ With recent advances in both surgical and medical therapy for lung cancer, and with the increasing use of CT imaging for screening, the number of survivors of early-stage lung cancer will likely increase. We sought to understand the patterns of recurrence and metachronous lung cancer, and the mode of their detection, after resection of early-stage non-small cell lung cancer (NSCLC) in patients who were followed by surveillance CT scan. Our objective was to characterize the patterns of recurrence and second primary lung cancers in patients with resected early-stage NSCLC and to assess the efficacy of a surveillance strategy using scheduled interval chest $\mathrm{CT}$ imaging alone. 


\section{Abbreviations and Acronyms}

$\mathrm{CT}=$ computed tomography

NSCLC $=$ non-small cell lung cancer

PET = positron emission tomography

\section{PATIENTS AND METHODS \\ Patient Cohort}

A retrospective review of a prospectively maintained database was performed for patients who were treated for lung cancer and followed at Memorial Sloan-Kettering Cancer Center between January 2004 and December 2009. Individuals who underwent complete surgical resection for early-stage (pathologic stage I-II) NSCLC were included. Staging was performed in accordance with the seventh edition of the AJCC Cancer Staging Manual. ${ }^{6}$ Exclusion criteria were small cell and carcinoid pathology, neoadjuvant therapy, incomplete resection, and previously treated lung cancer. Patients without follow-up information after the initial postoperative visit were not included. Events and outcomes were abstracted from the medical record. Event status (defined as the first diagnosis of either recurrence or second primary cancer) was censored at the last thoracic clinic followup. Vital status was confirmed using the Social Security Death Index. Follow-up was conducted until October 2011. The study was approved by the Memorial Sloan-Kettering Cancer Center institutional review board.

\section{Surveillance Protocol}

In general, postoperative lung cancer surveillance was performed in accordance with National Comprehensive Cancer Network guidelines. ${ }^{7}$ During the first 2 years after surgery, each patient received a physical examination, interval history, and chest/upper-abdominal CT scan with or without contrast, at least every 6 to 12 months. Bronchoscopy, serum markers, and positron emission tomography (PET) were not used in routine follow-up. Follow-up visits and surveillance CT scans were performed yearly thereafter. Although all clinic appointments were conducted at our institution, some imaging studies were performed at outside facilities. At each follow-up visit, all new studies were reviewed by the clinician. Patients were followed by their thoracic surgeon or an oncologist, a radiation oncologist, or a nurse practitioner trained in thoracic survivorship care.

\section{Recurrence and Second Primary Lung Cancer}

The principal end point was the development of a recurrence or second primary lung cancer. Data extracted included the type of recurrence (distant, regional, or local), the method of detection, and whether the event was detected at a scheduled clinic visit as part of routine surveillance or through an unscheduled visit outside of the follow-up protocol. The manner of detection-whether by scheduled CT scan, presentation of new symptoms, or other means - was defined as the modality that led to the initiation of further workup and diagnosis of treatment failure. Local recurrence was defined as any new lesion adjacent to a staple line, to the bronchial stump, or in the residual lobe (in cases of sublobar resection). Regional recurrences involved lymph node stations 1 to 14 or the ipsilateral lung. Distant metastasis consisted of extrathoracic disease, metastasis to the contralateral lung, pleural metastasis, pleural effusion, or pericardial effusion. Diagnosis of recurrence was confirmed by biopsy in most cases or by further imaging (eg, PET scan or brain magnetic resonance imaging) to support the clinical diagnosis and the decision to initiate treatment.

Second primary lung cancer was defined according to the criteria of Martini and Melamed. ${ }^{8}$ A new, distinct pulmonary malignancy was considered a second primary cancer if it fulfilled any 1 of the following 3 criteria: (1) histologic results different from those of the index tumor; (2) same histologic results as the index tumor but diagnosed 2 years after the primary tumor; or (3) same histologic results as the index tumor, diagnosed within
2 years of the primary tumor, but located in different lobes or segments, with no positive intervening lymph nodes and no evidence of metastasis.

\section{Statistical Analysis}

The time to treatment failure was calculated from the date of surgery to the date of diagnosis of the event, by use of the date of the pathologic diagnosis whenever possible. Survival was estimated using the Kaplan-Meier method. Patients who did not experience new events were censored at the time of the last clinic follow-up. Except for the first year, the rate of recurrence and the rate of second primary cancer were calculated within mutually exclusive 12-month intervals after surgery with curative intent. Each 12month rate was determined by dividing the number of events observed during that interval by the corresponding total follow-up time. Patients who died or were lost to follow-up within the first 3 months were excluded from the study. The recurrence rate for the first year reflected the rate observed between postoperative months 3 and 12. All patients who were still at risk of having the event at the beginning of the interval were included in the calculation. As a result, all rates are expressed as the number of events per 100 person-years. Survival was compared across groups by use of the nonparametric log-rank test. All statistical tests were 2 -sided. SAS version 9.3 (SAS Institute, Inc, Cary, NC) was used to perform all statistical analyses.

\section{RESULTS \\ Patient and Treatment Characteristics}

Of the 1445 patients with early-stage lung cancer who were resected during the study period, 27 died within 90 days after surgery and 140 had no follow-up information after the initial postoperative visit. Therefore, a total of 1294 subjects had sufficient data to be included in the study, representing $90.3 \%$ of all patients with early-stage lung cancer resected during the study period at our institution. The median length of follow-up among patients who did not experience recurrence or have second primary cancer was 35 months from the date of surgery (range, 4-92 months). Nine percent of the patients were followed for less than 1 year, $18 \%$ were followed for 2 to 3 years, $54 \%$ were followed for 3 to 5 years, and $20 \%$ were followed for more than 5 years. Baseline characteristics are listed in Table 1. The mean number of scheduled surveillance CT scans received per patient was 1.9 for year 1 and 1.5 for year 2, decreasing to 1.0 for year 6 and 0.8 for year 7 .

Survival after diagnosis of recurrences or second primary tumors was significantly worse for patients with recurrences than for patients with second primary tumors $(P<.001)$; 3 -year survival from the date of recurrence or metachronous lung cancer was $24 \%$ for those with recurrences and $67 \%$ for those with second primary tumors.

\section{Recurrence}

Recurrences developed in 1 in 5 patients (257 [20\%]) during the study period (Table 2). Of these patients, 67 (26\%) had locoregional disease alone; the remainder had only distant recurrences $(112[44 \%])$ or both distant and locoregional disease $(78[30 \%])$. The majority of recurrences (157 [61\%]) were detected by scheduled surveillance CT scan. Most of the remaining recurrences $(82[32 \%])$ were detected outside of the routine follow-up protocol, most 
TABLE 1. Baseline and treatment characteristics of 1294 patients with early-stage non-small cell lung cancer

\begin{tabular}{|c|c|}
\hline Characteristic & No. $(\%)$ \\
\hline Female sex & $752(58)$ \\
\hline Current or former smokers & $1098(85)$ \\
\hline Cigarette pack-y (mean \pm SD) & $39 \pm 34$ \\
\hline $\mathrm{FEV}_{1}(\mathrm{~L}$, mean $\pm \mathrm{SD})$ & $2 \pm 0.07$ \\
\hline $\mathrm{FEV}_{1}(\%$ predicted, mean $\pm \mathrm{SD})$ & $89 \pm 21$ \\
\hline Age $(y$, mean \pm SD) & $69 \pm 10$ \\
\hline \multicolumn{2}{|l|}{ Stage } \\
\hline IA & $760(59)$ \\
\hline IB & $319(25)$ \\
\hline IIA & $134(10)$ \\
\hline IIB & $81(6)$ \\
\hline \multicolumn{2}{|l|}{ Procedure } \\
\hline Lobectomy & $955(74)$ \\
\hline Wedge & $188(14)$ \\
\hline Segmentectomy & $111(9)$ \\
\hline Bilobectomy & $22(2)$ \\
\hline Pneumonectomy & $17(1)$ \\
\hline \multicolumn{2}{|l|}{ Adjuvant therapy } \\
\hline None & $1092(84)$ \\
\hline Chemotherapy & $192(15)$ \\
\hline Chemotherapy and radiation & $1(<1)$ \\
\hline Radiation & $1(<1)$ \\
\hline Unknown & $8(1)$ \\
\hline \multicolumn{2}{|l|}{ Pathologic diagnosis } \\
\hline Adenocarcinoma & $995(77)$ \\
\hline Squamous & $222(17)$ \\
\hline Large cell & $32(2)$ \\
\hline Other & $45(4)$ \\
\hline
\end{tabular}

$F E V_{l}$, Forced expiratory volume in 1 second; $S D$, standard deviation.

commonly as a result of the development of new symptoms. The most common symptoms included neurologic changes, bone pain, dyspnea, and hoarseness. When recurrences were analyzed by the type of relapse, surveillance CT scan detected $82 \%$ of locoregional-only recurrences $(n=55)$. In contrast, only $46 \%$ of distant-only recurrences $(n=51)$ were detected by surveillance CT scan. The majority of recurrences (215 [85\%]) were confirmed by biopsy. Approximately half of the patients with relapses (120 [47\%]) received systemic therapy with chemotherapy or chemoradiation. In contrast, $26 \%$ of patients $(n=68)$ received local therapy with radiation or surgery.

Approximately one third of recurrences (179 [71\%]) were diagnosed more than 2 years after surgery. The hazard rate for recurrence peaked during the second year (10 events per 100 person-years of follow-up) but remained elevated, at 7 and 6 events per 100 person-years during years 3 and 4, respectively (Figure 1). Only at postoperative year 5 did the recurrence rate decrease markedly, to 2 events per 100 person-years.

\section{Second Primary Lung Cancer}

Second primary lung cancer was detected in 91 patients (7\%) (Table 3). Scheduled surveillance CT scan detected
TABLE 2. Characteristics of 257 patients with recurrence

\begin{tabular}{lc}
\hline \multicolumn{1}{c}{ Characteristic } & No. $(\%)$ \\
\hline Recurrence location & \\
Locoregional & 143 \\
$\quad$ Regional lymph nodes & $69(48)$ \\
Same side & $62(43)$ \\
Staple line & $40(28)$ \\
Distant & 188 \\
Pleural disease & $67(36)$ \\
Contralateral lung & $44(23)$ \\
Brain & $36(19)$ \\
Liver & $22(12)$ \\
Bone & $17(9)$ \\
Chest wall & $15(8)$ \\
Adrenal & $15(7)$ \\
Detection method & \\
Surveillance CT & $157(61)$ \\
Symptoms & $81(32)$ \\
Other & $17(7)$ \\
Unknown & $2(1)$ \\
Treatment of recurrence & \\
Chemotherapy & $90(35)$ \\
RT & $37(14)$ \\
Chemo + RT & $30(12)$ \\
Surgery + RT & $18(7)$ \\
Surgery & $13(5)$ \\
Other & $14(5)$ \\
None & $17(7)$ \\
Unknown & $38(15)$ \\
\hline$C T$ Computed tomography; $R T$, radiation therapy. &
\end{tabular}

the majority of second primary lung cancers (85 [93\%]). Tissue confirmation of second primary malignancies was obtained for 90 cases $(99 \%)$. Most second primary malignancies $(77[85 \%])$ were in the same histologic category as the index tumor (ie, adenocarcinoma or squamous) but demonstrated different histologic patterns (eg, lepidic vs micropapillary). Almost all were diagnosed as early-stage cancers (stage I, 84 [92\%]; stage II, 4 [4\%]). Correspondingly, more than half of these patients were subsequently treated with surgery $(55[60 \%])$. Wedge resection was the most common procedure performed $(46[82 \%])$. Unlike that for recurrences, the hazard rate for second primary cancer did not diminish after surgery, but instead increased over time, from 3 events per 100 person-years in year 2 to 6 events per 100 person-years in year 5 (Figure 1).

\section{False-Positive Results From Surveillance Computed Tomography Scans}

In addition to the 157 patients who had recurrences detected by surveillance CT scan, 329 asymptomatic patients $(25 \%)$ had abnormal or indeterminate surveillance CT scan results and underwent additional testing that did not result in a diagnosis of recurrence or second primary cancer. The most common noninvasive test performed was repeat short-interval CT scan within 2 to 3 months of the incident 


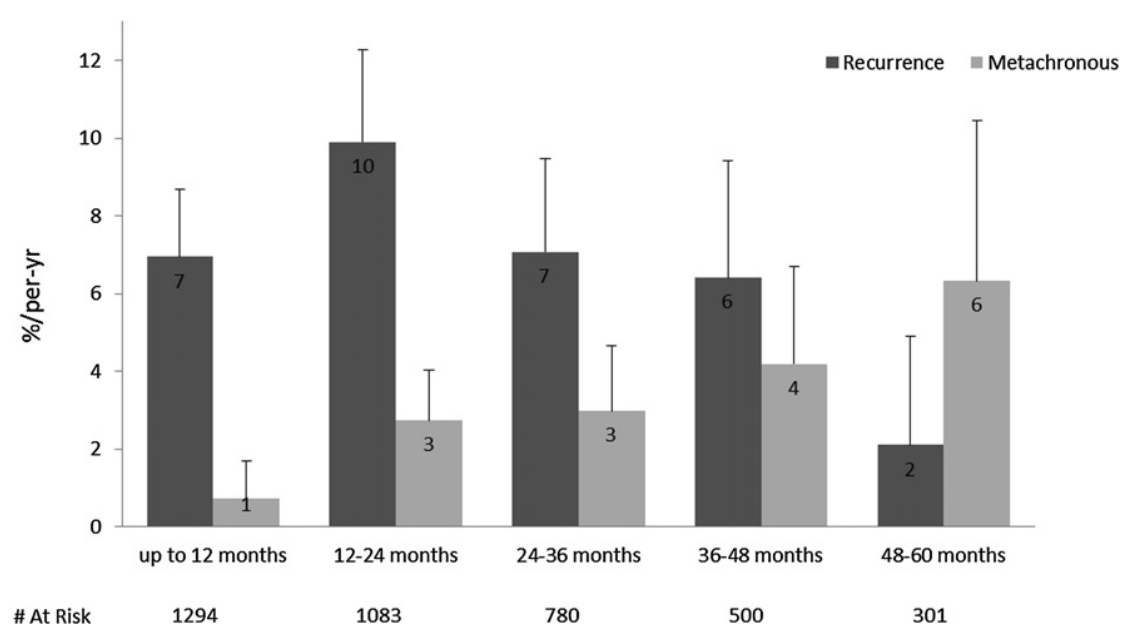

FIGURE 1. Hazard rates of recurrent and second primary lung cancer after resection over time.

scan $(275[21 \%])$ (Table 4). Invasive testing was performed in 61 patients $(5 \%)$. CT-guided needle biopsy was the most frequent invasive procedure, performed in 41 patients $(3 \%)$. Among the 329 patients who received false-positive

TABLE 3. Characteristics of 91 patients with second primary lung cancer

\begin{tabular}{lc}
\hline \multicolumn{1}{c}{ Characteristic } & No. $(\%)$ \\
\hline Detection method & \\
Surveillance CT & $85(93)$ \\
Symptoms & $2(2)$ \\
Other & $2(2)$ \\
Unknown & $3(3)$ \\
Stage of second primary & \\
IA & $73(80)$ \\
IB & $11(12)$ \\
IIA & $1(1)$ \\
IIB & $3(3)$ \\
IIIA & $3(3)$ \\
Pathologic diagnosis & \\
Adenocarcinoma & $72(79)$ \\
Squamous & $13(14)$ \\
Small cell & $3(3)$ \\
Large cell & $2(2)$ \\
Treatment & \\
Surgery & $51(56)$ \\
RT & $23(25)$ \\
Chemotherapy & $3(3)$ \\
Other & $9(10)$ \\
None & $1(1)$ \\
Unknown & $4(4)$ \\
Resection & \\
Wedge & $46(82)$ \\
Lobectomy & $6(11)$ \\
Segmentectomy & $3(5)$ \\
Pneumonectomy & $1(2)$ \\
\hline$C T$, Computed tomography $R T$, radiation therapy. \\
\end{tabular}

surveillance CT scan results, a total of 4 complications resulted from subsequent invasive procedures, for a $0.3 \%$ risk of complications resulting from ultimately unnecessary invasive procedures ( 3 individuals developed pneumothoraces from CT-guided lung biopsies and required hospitalization, and 1 patient experienced persistent air leak and empyema after an open biopsy). No deaths resulted from these complications. In addition, 14 nonlung malignancies, either primary or metastatic, were diagnosed incidentally during the course of routine CT surveillance.

\section{DISCUSSION}

We examined the utility of CT surveillance in the follow-up care of 1294 resected early-stage lung cancer survivors and characterized their patterns of recurrence and second primary lung cancer. Although the majority of patients in this cohort had stage I disease, we still observed new events in more

TABLE 4. Tests performed as a result of false-positive surveillance computed tomography scan results

\begin{tabular}{lc}
\hline \multicolumn{1}{c}{ Modality } & No. $(\%)$ \\
\hline Noninvasive & \\
Short-interval CT scan & $275(21)$ \\
PET & $70(5)$ \\
US & $8(<1)$ \\
Bone scan & $3(<1)$ \\
Chest X-ray & $1(<1)$ \\
Invasive & \\
CT-guided biopsy & $41(3)$ \\
Bronchoscopy/EBUS & $16(1)$ \\
Open biopsy & $4(<1)$ \\
Thoracentesis & $4(<1)$ \\
Mediastinoscopy & $3(<1)$ \\
\hline CT, Computed tomography; PET, positron emission tomography; $U S$, ultrasound; \\
EBUS, endobronchial ultrasound.
\end{tabular}


than one quarter of patients, with $20 \%$ developing recurrent disease and another 7\% developing second primary lung cancer. Despite the conventional wisdom that recurrences occur during the first 2 years after resection, we found that a high risk of recurrence persisted for up to 4 years after resection. CT surveillance was successful in detecting more than $90 \%$ of the second primary cancers and approximately two thirds of the recurrences diagnosed during the study period. After the first year, metachronous lung cancers developed at a rate of $3 \%$ to $6 \%$ per person-year, higher than the rate of $1 \%$ to $3 \%$ previously reported in other series. Indeterminate or false-positive results led to additional testing in one quarter of the patients. Five percent of all patients underwent invasive procedures as the consequence of a false-positive surveillance test result, but a major complication developed in only $0.3 \%$ (4 patients). To our knowledge, this is the largest series to date on recurrence and new primary lung cancer development in patients followed by surveillance CT scan.

The detection rate of early-stage lung cancer has increased with the increasing use of cross-sectional imaging, such as CT scanning, in the diagnostic armamentarium of health care providers. Advances in surgical technique and minimally invasive technology have led to the widespread adoption of video-assisted thoracoscopic approaches to anatomic resection for the treatment of early-stage lung cancers. The resulting confluence of these factors has led to a growing contingent of patients undergoing surgery for early-stage cancers and the recognition of the need to define better standards of care for lung cancer survivors. The National Lung Screening Trial convincingly demonstrated that CT screening of high-risk patients significantly reduced lung cancer mortality. ${ }^{2}$ One could argue that a patient with a recent history of lung cancer also could be considered to be at high risk, raising the question of whether a similar utility of CT screening would be observed among lung cancer survivors.

Although several organizations have recommended various guidelines that include CT scanning for follow-up after curative treatment for lung cancer, there is no consensus, highlighting a lack of clarity on the issue..$^{3,7}$ Furthermore, although most authors advocate some form of imaging surveillance, others have previously suggested a follow-up protocol based on symptoms alone, citing the failure of routine surveillance imaging to detect recurrences in asymptomatic patients. ${ }^{5}$ In a study by Pairolero and colleagues, ${ }^{9}$ patients treated for stage I NSCLC were closely followed by history, physical examination, and chest $\mathrm{x}$-ray, as well as by blood, urine, and sputum studies. However, $41 \%$ of recurrences were still detected outside of scheduled visits. Because $53 \%$ of recurrences were symptomatic, the authors reported that symptoms, not scheduled tests, were the most sensitive recurrence detection method. The outcomes of CT surveillance have been reported in several series. ${ }^{4,10,11}$ Chiu and colleagues ${ }^{10}$ reported that surveillance standard-dose $\mathrm{CT}$ after curative resection was more sensitive than low-dose CT and chest x-ray. In a decision analysis by Kent and colleagues, ${ }^{12}$ the cost of surveillance CT was estimated to be $\$ 47,676$ per quality-adjusted life-year gained. Age more than 65 years, cost of CT more than $\$ 700$, incidence of second primary lung cancer less than $1.6 \%$ per person-year, and false-positive rate of more than $14 \%$ made CT surveillance cost-ineffective.

In contrast, other authors have recommended more aggressive surveillance programs that combine CT screening with routine bronchoscopy. ${ }^{11}$ In one study of 192 subjects treated for NSCLC, the authors reported that an intensive surveillance program using routine bronchoscopy and thoracic/abdominal CT scan was feasible and that it detected a significant number $(38 \%)$ of recurrences in asymptomatic patients.

\section{Recurrence}

Recurrences developed in 1 of 5 patients with early-stage lung cancer in the present study and were detected by surveillance CT scan in more than $60 \%$ of cases. However, for approximately one third of patients, the development of symptoms prompted the diagnosis of recurrence during the interval between surveillance scans. The majority of recurrences involved metastatic disease to distant sites (Table 2) and were treated correspondingly, with systemic therapy. Locoregional recurrences alone occurred in one quarter of patients, including in the ipsilateral lung or regional lymph nodes, which were potentially amenable to local therapy. The risk of recurrence reached a plateau at 2 years but remained elevated for up to 4 years, before declining. A recent analysis of the recurrence dynamics in 1506 patients with stage I to IIIA NSCLC who underwent resection between 1995 and 2008 similarly demonstrated elevated hazard rates of recurrence for up to 4 years after surgery. ${ }^{10}$ Moreover, in a long-term follow-up of 285 patients after surgery for stage I NSCLC, Murthy and colleagues ${ }^{13}$ used parametric modeling to develop a multiphase hazard model. They reported an early hazard phase in the first 5 years after surgery, followed by a constant late phase thereafter. However, in contrast to our analysis, only tumors with different histology were considered metachronous malignancies.

\section{Second Primary Lung Cancer}

Second primary cancers developed less frequently than recurrences did. In a retrospective series by Lamont and colleagues, ${ }^{4} 124$ patients underwent surveillance chest $\mathrm{x}$-rays and CT scans. Up to $58 \%$ and $37 \%$ of second primary tumors were detected by $\mathrm{CT}$ and chest $\mathrm{x}$-ray, respectively. Approximately $68 \%$ of patients benefited from surgical resection. In our study, CT surveillance was able to detect more than $90 \%$ of second primaries. As expected, the majority of these cancers were detected at an early stage, and their treatment correspondingly centered on local 
therapy, with surgical resection as the predominant option. Our observed rate of second primary cancers was higher than the rate of $1 \%$ to $3 \%$ per patient-year reported in previous studies. ${ }^{14}$ The increased proportion of adenocarcinoma relative to squamous cell histologic profile may predispose our patient population to multifocal or second primary cancers, compared with the populations in older studies. The inclusion of patients with more advanced disease in other studies may have introduced the competing risk of higher recurrence rates and lower survival, thereby decreasing the observed number of second primary cancers. Finally, studies in which routine surveillance CT scan was not performed may have mistaken advanced second primary tumors as recurrences from the index tumors. ${ }^{8,15}$

\section{Potential Risks of Computed Tomography \\ Surveillance}

With any diagnostic test comes the risk of false-positive results and their unintended consequences. False-positive results often lead to further testing, which can involve invasive procedures or increased exposure to radiation, while increasing patient anxiety and costs. Postoperative changes in the resected patient may complicate the interpretation of radiographic abnormalities and lead to a higher incidence of false-positive results. One quarter of the patients in our cohort required additional workup — because of abnormalities found on surveillance CT scans-that ultimately failed to identify any evidence of recurrent malignancy or second primary lung cancer. Only $5 \%$ of patients underwent what could be considered unnecessary invasive procedures for false-positive or indeterminate surveillance scan results; CT-guided needle biopsy was the most common such procedure. Of importance, only $0.3 \%$ of the patients in our cohort experienced a complication unnecessarily for what was ultimately a false-positive surveillance scan result. The decision to act on a CT scan read as abnormal is highly operator-dependent and relies on the clinical judgment of the clinician. One study reported that only $29 \%$ of all surveillance $\mathrm{CT}$ scans reported as abnormal by the radiologist were actually considered suspicious enough by the clinician to warrant additional testing. ${ }^{12}$ Korst and colleagues ${ }^{16}$ reported that pleural effusion and pulmonary nodules greater than $1 \mathrm{~cm}$ or an increase in size correlated with recurrence. Defining our false-positive rate with respect to the clinical context, rather than simply including all scans reported as abnormal on the radiology report, affords a more accurate estimate of the true risks of postoperative surveillance.

\section{Implications for Surveillance}

The use of routine surveillance CT imaging is important in the follow-up care of early-stage lung cancer survivors. The risk of second primary lung cancer remains high after definitive resection. Surveillance CT scan detected more than $90 \%$ of second primary lung cancers at an early stage, when definitive local therapy was possible for most patients. Likewise, surveillance CT scan detected the majority of new recurrences before the onset of symptoms. Several randomized control trials have demonstrated a survival benefit with chemotherapy, compared with supportive care, in patients with advanced lung cancer. ${ }^{13}$ Given that the observed rate of recurrence remained elevated during the first 4 years after surgery, it may be beneficial to maintain close-interval follow-up throughout this period.

The concept of surveillance presumes that there is a benefit from earlier detection. At present, no randomized prospective study has been performed to evaluate the outcomes of regimens with different surveillance intensities. One meta-analysis of postoperative surveillance found a trend toward improved survival among patients who received intensive follow-up. ${ }^{14}$ However, the analysis included studies with small sample sizes and different definitions of intensive follow-up. The value of screening for second primary cancers seems plausible in light of the results of the National Lung Screening Trial, but it remains less clear whether earlier detection of distant recurrences can lead to improved survival or quality of life. Further understanding will depend on randomized, controlled trials to assess survival and patient-centered outcomes. At present, one such study is under way. ${ }^{12}$ The IFCT-0302 trial is a phase III, multicentric, randomized, controlled trial that aims to study the benefit of surveillance $\mathrm{CT}$ and fiberoptic bronchoscopy after complete resection for stage I to IIIa NSCLC. In addition to attending scheduled clinic visits, patients are randomized to receive chest X-ray or chest X-ray, CT scan, and fiberoptic bronchoscopy. Chest CT scan is the most commonly recommended surveillance test in most guidelines. Nonetheless, the majority of recurrences in this patient population are distant, and most are extrathoracic. PET-CT is now commonly used in preoperative staging of lung cancer. In a small series, postresection surveillance using PET-CT identified recurrences that were missed by chest CT. ${ }^{17}$ However, PET-CT was less sensitive than CT in the detection of small adenocarcinomas and groundglass lesions. At present, its role in surveillance after surgical resection remains to be defined. Another important unexplored aspect of CT surveillance is the benefit of a regimen tailored to patient characteristics, such as age, comorbidities, and extent of resection. An individualized approach to follow-up should be evaluated in future studies.

\section{Study Limitations}

There are several limitations to the present study, including those inherent in retrospective analyses. This was a single-institution experience, albeit large, and it may not be generalizable to other cohorts. The study cohort was limited to patients followed in our institution; those followed elsewhere were not included. The patterns of recurrence and second primary lung cancer can be affected by 
competing events, such as death from other causes. Such analyses were precluded by the lack of information on the cause of death for all patients. Furthermore, the definition of second primary lung cancer remains controversial. Although the criteria of Martini and Melamed ${ }^{8}$ are the most widely used, they may miscategorize some tumors, leading to inaccuracies in rates of recurrence and second primary lung cancer. However, it is still crucial to distinguish second primary tumors from relapse in clinical practice because of the significant differences in their treatment and prognosis. Last, surveillance scans were performed at predefined intervals. Recurrences may develop in between CT scans and clinic appointments. Interval censoring may lead to an overestimation of time to event in our analysis.

\section{CONCLUSIONS}

Routine CT surveillance detected the majority of recurrences, as well as the majority of second primary lung cancers. We found that the rate of recurrence remained elevated until 4 years after surgery, which is in contrast to the conventional wisdom. False-positive surveillance scan results led to subsequent invasive procedures for only $5 \%$ of patients. Most of the second primary lung cancers were detected at early stages, and more than half of patients underwent surgical resection. On the basis of our results, we support the use of CT surveillance for survivors of earlystage NSCLC. However, the optimal interval between surveillance scans remains subject to further study.

\section{References}

1. Jemal A, Siegel R, Xu J, Ward E. Cancer statistics, 2010. CA Cancer J Clin. 2010;60:277-300.

2. National Lung Screening Trial Research Team, Aberle DR, Adams AM, Berg CD, Black WC, Clapp JD, Fagerstrom RM, et al. Reduced lung-cancer mortality with low-dose computed tomographic screening. N Engl J Med. 2011;365:395-409.

3. Rubins J, Unger M, Colice GL. Follow-up and surveillance of the lung cancer patient following curative intent therapy. Chest. 2007;132:355s-67s

4. Lamont JP, Kakuda JT, Smith D, Wagman LD, Grannis FW Jr. Systematic postoperative radiologic follow-up in patients with non-small cell lung cancer for detecting second primary lung cancer in stage IA. Arch Surg. 2002;137:935-9.

5. Walsh GL, O'Connor M, Willis KM, Milas M, Wong RS, Nesbitt JC, et al. Is follow-up of lung cancer patients after resection medically indicated and cost-effective? Ann Thorac Surg. 1995;60:1563-72.

6. Edge SB, Byrd DR, Compton CC, Fritz AG, Greene FL, Trotti A. AJCC Cancer Staging Manual. 7th ed. New York: Springer-Verlag; 2009

7. National Comprehensive Cancer Network. Guidelines for surveillance following therapy for NSCLC. Available at: www.nccn.org. Accessed October 4, 2010.

8. Martini N, Melamed MR. Multiple primary lung cancers. J Thorac Cardiovasc Surg. 1975;70:606-12.

9. Pairolero PC, Williams DE, Bergstrahl EJ, Piehler JM, Bernatz PE, Payne WS. Postsurgical stage I bronchogenic carcinoma: morbid implications of recurrent disease. Ann Thorac Surg. 1984;38:331-6.

10. Chiu CH, Chern MS, Wu MH, Hsu WH, Wu YC, Huang MH, et al. Usefulness of low-dose spiral CT of the chest in regular follow-up of postoperative non-small cell lung cancer patients: preliminary report. J Thorac Cardiovasc Surg. 2003; 125:1300-5.

11. Westeel V, Choma D, Clément F, Woronoff-Lemsi MC, Pugin JF, Dubiez A, et al. Relevance of an intensive postoperative follow-up after surgery for non-small cell lung cancer. Ann Thorac Surg. 2000;70:1185-90.

12. Kent M, Korn P, Port JL, Lee PC, Altorki NK, Korst RJ. Cost effectiveness of chest computed tomography after cancer resection: a decision analysis model. Ann Thorac Surg. 2005;80:1215-23.
13. Murthy SC, Reznik SI, Ogwudu UC, Farver CF, Arrossi A, Batizy LH, et al. Winning the battle, losing the war: the noncurative "curative" resection for stage I adenocarcinoma of the lung. Ann Thorac Surg. 2010;90:1067-74.

14. Johnson B. Second lung cancers in patients after treatment for an initial lung cancer. J Natl Cancer Inst. 1998;90:1335-45.

15. Antakli T, Schaefer EF, Rutherford JE, Read RC. Second primary lung cancer. Ann Thorac Surg. 1995;59:863-7.

16. Korst RJ, Kansler AL, Port J, Lee PC, Altorki NK. Accuracy of surveillance computed tomography in detecting recurrent or new primary lung cancer in patients with resected lung cancer. Ann Thorac Surg. 2006;82:1009-13.

17. Choi S, Kim YT, Kim SK, Kang KW, Goo JM, Kang CH. Positron emission tomography-computed tomography for postoperative surveillance in non-small cell lung cancer. Ann Thorac Surg. 2011;92:1826-32.

\section{Discussion}

Dr Sean C. Grondin (Calgar, Alberta, Canada). This is an important article for thoracic surgeons. Many organizations are publishing guidelines on CT screening, as you mentioned, and it is a hot topic for discussion. There are a lot of resources at stake, so I appreciate your group trying to tackle this important question.

I have just a few questions for you. Many patients undergo CT for follow-up of a nodule, but they are concerned about the radiation exposure. I noticed in your protocol, the first 2 years you were doing CT scans with contrast and then after that it was CT scans without contrast. Can you explain to me the justification for using contrast in the first 2 years? This approach contradicts other CT screening guidelines by other organizations.

Dr Lou. You mean the justification for using contrast in the first 2 years?

Dr Grondin. Yes, most good CT radiologists will tell you that you probably do not need contrast and that a CT without contrast is good for screening, so why would you need contrast?

Dr Lou. We used CT with contrast in the first 2 years because it may be more effective in identification of hilar or mediastinal lymph node metastases, as well as liver metastases. However, we do recognize that the effectiveness of contrast versus noncontrast CT has not been adequately evaluated in lung cancer surveillance. In fact, the most recent National Comprehensive Cancer Network recommendations were changed from $\mathrm{CT}$ with contrast for the first 2 years to CT with or without contrast. The use of contrast in follow-up essentially may make logical sense, but this has not been validated by large prospective data.

Dr Grondin. Fair enough. I noticed your recurrence rate is higher than what you would expect from historical series, $6 \%$ to $10 \%$. Can you explain to me why that might be in your series? Is this a reflection of the staging of the patients before they underwent operation, they were not fully staged, or why is this higher in your group do you think?

Dr Lou. I am not sure if the recurrence rate found in our group was actually higher than in other series. The traditionally quoted 5 -year recurrence rate of stage I disease is $20 \%$ to $39 \%$. We found that $20 \%$ of patients developed recurrences. But one thing we did that was different was that in addition to looking at only the proportion of recurrence over time, we looked at the rate of recurrence-meaning events over the number of months followed within each 12-month interval, and that is the recurrence rate we found.

Dr Grondin. The next question I have pertains to imaging of patients for follow-up. If we all agree that CT is pretty good, and down deep in my heart I think that CT probably is better than chest 
$\mathrm{x}$-ray at following patients postoperatively, do you think that might be even better than CT?

Dr Lou. Although PET scan has not been part of any of the organizations' guidelines for surveillance, it recently has been investigated by some groups in Korea and the United States. The last study that I found actually gave patients both CT scan and PET surveillance, and they found that the PET scan was able to detect a few percentages more of recurrences, most of which were distal. However, when compared with CT scans, PET scans also missed a few cases, especially when these masses are localized to the lung if they were small or ground-glass opacities. So there are pluses and minuses of PET scans, and I think more studies are warranted in this area. But one must be cautious in interpreting these studies, because the question of which is the superior modality should be the actual effect on survival and patient-oriented outcomes instead of only slight improvement in detection sensitivity.

Dr Grondin. My last question has to do with survival. That is the elephant in the room. It is great to do imaging studies such as CT, but at the end of the day is there any improvement in survival? I noticed that approximately three quarters of your patients had distant metastases detected by CT. I am not aware of any data that treating this subgroup of patients significantly extends survival. What are your thoughts on whether this early detection of recurrent disease with CT will translate into a survival advantage?

Dr Lou. This is something we thought of extensively as a group. Again, there have been no prospective randomized trials looking at the exact effect of earlier detection by surveillance programs. However, at least for second lung primaries from our studies and some previous series, you can see that most of them were able to be detected in the early stage when curative therapies could be given. It could be inferred that this kind of early detection would lead to a survival benefit in those with a second primary tumor, but again prospective verification is needed. However, it is more controversial when you are talking about recurrences because most patients with recurrences can only receive palliative therapy, so the question of whether that kind of therapy would be more beneficial if given a few months or weeks before would actually affect long-term survival is more controversial and definitely needs to be studied. We should not make a judgment on that before prospective studies are done.

Dr Grondin. Thank you, and great job.
Dr Robert Shen (Rochester, Minn). I noted that $25 \%$ of patients with a second primary lung cancer were treated with external beam radiation therapy rather than surgical resection. Do you have a sense of how many of those patients were medically ineligible or thought to be medically ineligible for further surgery or were offered surgery and refused? A corollary to that is what is your institutional algorithm for a patient who is thought to have a second primary? Is the patient offered surgery as the first treatment or is it thought to be equivalent to offer stereotactic radiation?

Dr Lou. With regard to second primary lung cancers, it is always interesting to look at the outcomes and their treatments. We do not have the exact numbers and the exact reasons why these patients received surgery versus another treatment; however, I would suspect a lot of these patients having undergone one surgical resection already may have limited physiologic and respiratory reserve, which may limit their ability to undergo surgery, but that is just a general sense of what I think happened. In terms of how the decision was made exactly in each case, it was most likely dependent on each radiologist and surgeon, but it is our institution's practice that whenever a second primary lung cancer is detected, the best alternative should be surgical resection if tolerated.

Dr Frederic Grannis (Duarte, Calif). This is the second of 2 important articles presented during this meeting on metachronous lung cancer, and, although they are single institution reviews, when those single institutions are Mayo Clinic and Memorial Sloan Kettering, people have to pay close attention. I think yours is the stronger study because it relies on a defined diagnostic regimen, and I think the evidence is stronger, but I have a problem. Your second author, Dr Peter Bach, authored an article in The Journal of the American Medical Association last month in which he published lung cancer screening guidelines for the American College of Chest Physicians and American Society of Clinical Oncology. In those guidelines he ignored his own data presented today by omitting this important ultra-high risk group of people from lung cancer screening guidelines. I think this will have adverse consequences and that lives will be lost because of that omission. So when you return to New York City, please ask Dr Bach why he omitted his own data in writing the American College of Chest Physicians and American Society of Clinical Oncology guidelines.

Dr Lou. Thank you. 